\title{
Arbeit in den Gettos: Rettung oder temporärer Vernichtungsaufschub?
}

War Arbeit für die von den Nationalsozialisten in Gettos eingesperrten Juden gleichbedeutend mit Überleben? Der Judenälteste von Litzmannstadt - diesen Namen hatten die deutschen Besatzer der Stadt Lodz oder Łódź im Frühjahr 1940 gegeben - ging davon aus, dass dies so war. Litzmannstadt ist das wohl prominenteste Beispiel einer in großem Ausmaß betriebenen Gettoindustrie und damit verbunden dem Einsatz weiter Teile der Bevölkerung in den Werkstätten und Fabriken. Mordechai Chaim Rumkowski hielt am 4. Januar 1942 vor Mitgliedern seiner Verwaltung und anderen ausgewählten Zuhörern eine Rede. Knapp zwei Wochen, bevor die Deportationen Zehntausender Lodzer Juden in das Vernichtungslager Kulmhof (Chełmno) ${ }^{1}$ begannen, blickt der von der deutschen Verwaltung für die Organisation des Gettolebens verantwortlich gemachte Rumkowski zunächst zurück:

\begin{abstract}
Denke ich zurück, dann erfüllt mich in erster Linie der fürwahr rekordartige Ausbau des Gettos zu einem Arbeitszentrum mit Stolz. Buchstäblich aus dem Nichts haben wir doch enorme Arbeitsstätten errichtet, die verschiedensten Werkstätten und Fabriken in Betrieb genommen. Schon heute beschäftigen wir eine Armee von nahezu 50000 Menschen. Mit solch einer Zahl an Arbeitenden müssen alle ernsthaft rechnen. Und damit rechnen auch alle, bis in die entscheidenden Instanzen hinein. [...] Ich bin mir sicher, dass die Behörden zu keinen Schikanen greifen werden, solange das Getto ehrlich und gut arbeitet. [...] DER PLAN ZU BEGINN DES NEUEN JAHRES: Dieser Plan ist: ARBEIT, ARBEIT und noch einmal ARBEIT. Mit eisernem Willen werde ich danach streben, dass sich für alle im Getto eine Arbeit findet [...]. Wenn ich dieses Programm verwirkliche, kann ich aufgrund der unanfechtbaren Zahlen beweisen, dass die Juden im Getto ein produktives Element und sie daher wertvolle Menschen sind. ${ }^{2}$
\end{abstract}

Der Gedanke, dass Juden sich durch ihre Produktivität retten würden, war keineswegs abwegig, in zahlreichen Gettos wurde von den verantwortlichen jüdischen Funktionären entsprechend gehandelt. Oftmals traf sich dieser Wille,

1 Deutsche Lager wie z.B. das Vernichtungslager Belzec und das Zwangsarbeits- bzw. Konzentrationslager Plaszow gebe ich hier in der zeitgenössischen deutschen Schreibweise wieder, da es nicht um die polnischen Ortschaften Bełżec und Płaszów, sondern um die von Deutschen eingerichteten Orte des Terrors geht. Und so verwende ich in der Folge auch den deutschen Namen für die Vernichtungsstätte Kulmhof.

2 Zit. n.: Die Chronik des Gettos Lodz/Litzmannstadt 1942 (künftig: Chronik 1942), hrsg. v. Sascha Feuchert et al., Göttingen 2007, S. 23-27. 
Arbeit $\mathrm{zu}$ organisieren, mit dem Profitstreben deutscher Verantwortlicher auch hier ist Litzmannstadt mit dem Leiter der deutschen Gettoverwaltung Hans Biebow ein gutes Beispiel - oder auch Firmeninhaber, die die günstige Arbeitskraft der im Getto eingesperrten Juden ausnutzten. Es gab dabei verschiedene Arten der Organisation von Gettoarbeit: In Litzmannstadt war alles zentral geregelt und die Arbeiter blieben im Getto, wo sie in Werkstätten arbeiteten. In vielen anderen Gettos verließen Teile der Arbeiter morgens unter Bewachung das Getto, um außerhalb zu verschiedenen Arbeiten eingesetzt zu werden. Mitunter waren Juden sogar eine Zeit lang bei demselben Arbeitgeber beschäftigt wie vor dem Krieg. Insgesamt gilt: Menschen in den Gettos bemühten sich aktiv darum, eine Arbeitsstelle $\mathrm{zu}$ finden. Nur dies garantierte ihre - wenngleich dürftige - Ernährung, später aber war eine Arbeitskarte die nahezu einzige Möglichkeit, den Deportationszügen in die Vernichtung zu entgehen.

Bedeutete Arbeit aber wirklich Rettung oder nur einen Aufschub, bevor die Menschen doch ermordet wurden? In diesem Artikel soll an den Beispielen der Gettos Litzmannstadt im Reichsgau Wartheland und Krakau im Generalgouvernement aufgezeigt werden, welche Bedeutung Arbeit in den Gettos für die Bewohner hatte und wie sie organisiert war. Nicht geleistet wird eine Geschichte dieser Gettos oder gar eine Darstellung der Gettoisierung im nationalsozialistisch besetzten Osteuropa insgesamt.

Laut den Ergebnissen des USHMM gab es im deutsch beherrschten Ostund Ostmitteleuropa etwa 1100 bis 1200 Gettos, davon existierten einige nur über einen sehr kurzen Zeitraum. Im Gebiet von Polen in den Grenzen von 1939 etwa 600, im Baltikum etwa 130, in der Sowjetunion etwa 250 unter deutscher Besatzung, weitere Gettos gab es unter rumänischer Herrschaft in Transnistrien und in Groß-Ungarn. Die Unterschiede zwischen den Gettos waren teilweise gravierend. Es existierten geschlossene Gettos, die durch einen Zaun oder - seltener - eine Mauer streng abgeriegelt waren, daneben aber auch sog. offene Gettos, die nicht äußerlich begrenzt waren oder sogar einfach aus einer bestimmten festgelegten Straße im Ort bestanden. Der Grad der Isolation war recht unterschiedlich, außerdem existierten die Gettos auch unterschiedlich lang. ${ }^{3}$ Und so differierten auch die Organisation und die Bedingungen von Arbeit erheblich. ${ }^{4}$

3 Martin Dean (Hrsg.), Encyclopedia of Camps and Ghettos 1933-1945, Bd. II.: Ghettos in German-Occupied Eastern Europe, Bloomington 2011. Siehe auch: The Yad Vashem Enzyclopedia of the Ghettos During the Holocaust, hrsg. v. Guy Miron/Shlomit Shulhani, 2Bde., Jerusalem 2009; BGN 25 (2009).

4 Siehe dazu jetzt die Beiträge in: Jürgen Hensel/Stephan Lehnstaedt (Hrsg.), Arbeit in den nationalsozialistischen Ghettos, Osnabrück 2013. 


\section{Die Organisation der Arbeit}

In Litzmannstadt organisierte Mordechai Chaim Rumkowski seit dem Frühjahr 1940, die Arbeit im Getto. ${ }^{5}$ Aus nahezu jeder Branche gab es im Getto Spezialisten. Bereits am 1. Mai 1940 wurde die erste Schneidereiabteilung gegründet und die Registrierung aller Schneider begann. Großes Improvisationstalent war gefordert: Die jeweiligen Fachleute wurden aufgerufen, sich zu melden, kamen zur Arbeit, brachten mit, was sie an Werkzeugen oder Maschinen hatten retten können, und die Produktion begann. Viele Maschinen fehlten anfangs, auch der Mangel an Rohstoffen war ein großes Problem. Anfang Oktober 1940 existierten dennoch bereits Fabriken in 18 verschiedenen Branchen, so gab es Schneidereien, Kürschnereien, Strickereien und Gerbereien, eine Gummi-Fabrik und eine Tapeziererei. $^{6}$

Das Zentralbüro der Arbeits-Ressorts - Ressort wurden im Getto die Fabriken genannt ${ }^{7}$ - war für die Organisation der Werkstätten und Fabriken zuständig. Unter seinem Leiter Aron Jakubowicz fungierte es seit dem 1. Oktober 1940 als Mittlerinstanz zwischen der deutschen Gettoverwaltung und den einzelnen Betrieben im Getto bei der Ausführung von Aufträgen. Die Bestellungen der Kunden gingen über die Gettoverwaltung an dieses Büro, welches das betreffende Ressort damit beauftragte sowie die Einhaltung des Liefertermins und die Qualität der Ware kontrollierte. Größter Abnehmer war mit etwa 90 Prozent des Bestellvolumens das Deutsche Reich und hier vor allem die Wehrmacht, aber auch die Stadtverwaltung Litzmannstadt. Etwa zehn Prozent der Bestellungen kamen von Privatfirmen wie Neckermann, dem Alsterhaus Hamburg, der AEG

5 Zur Arbeit in den kleineren Gettos des Warthegaus siehe: Stephan Lehnstaedt, Jewish Labor in the Smaller Ghettos in the Warthegau Region, in: YVS 38 (2010), S. 47-84; Andrea Löw, Warthegau. „Und diese Stadt wird leben, weil sie so leidenschaftlich leben will“, in: Hensel/Lehnstaedt, Arbeit, S. 113-137. In diesem Artikel habe ich auch die Arbeitsbedingungen im Getto Litzmannstadt genauer dargestellt und konnte mich hier darauf stützen.

$6 \mathrm{Zu}$ den unterschiedlichen Branchen im Getto der Bericht eines Überlebenden: Bendet Hershkovitch, The Ghetto in Litzmannstadt (Lodz), in:YIVO 5 (1950), S. 85-122. Ich stütze mich bei diesen Ausführungen in hohem Maße auf: Andrea Löw, Juden im Getto Litzmannstadt. Lebensbedingungen, Selbstwahrnehmung, Verhalten, Göttingen 2006.

7 Das Wort Ressort bürgerte sich schon früh in der Gettosprache ein und bald wurden alle Betriebe des Gettos mit der Bezeichnung Ressort versehen. Es hieß also Schneiderressort oder Tischlerressort. Das aus dem Französischen stammende Wort wurde laut eines Eintrags von Oskar Singer für die 1944 im Getto verfassten Enzyklopädie - mit dem „t“ am Ende gesprochen, in: Archiwum Żydowskiego Instytutu Historycznego (Archiv des Jüdischen Historischen Instituts in Warschau, künftig: AŻıH), 205/311, Bl. 323. 
oder Telefunken. ${ }^{8}$ Die Löhne, die den Auftraggebern in Rechnung gestellt wurden, gingen auf das Konto der Gettoverwaltung, von dem die Versorgung des Gettos finanziert wurde. In der eigenen Währung des Gettos bekamen die Arbeiter von der jüdischen Verwaltung ihre Löhne ausbezahlt.

Zumindest eine Zeit lang konnte ein Arbeiter im Getto Litzmannstadt seine Familie dadurch, dass er angestellt war, miternähren: Es ist nicht ganz klar, wann dieses System eingeführt wurde, doch berichtet die im Archiv des Gettos zwischen Januar 1941 und Juli 1944 verfasste Tageschronik im Mai 1942 von der Abschaffung von Familienzulagen: In den Monaten zuvor hatten nicht beschäftigte Familienmitglieder eines Arbeiters je 15 Mark monatlich als Zulage erhalten. ${ }^{9}$ Ein weiterer Faktor, der die Arbeit für die Menschen im Getto so wichtig machte, war die Verpflegung am Arbeitsplatz: Mittags gab es eine Suppe, manchmal bekamen die Arbeiter weitere Verpflegung, teilweise konnten sie davon sogar etwas aus der Fabrik hinausschmuggeln und ihre Familie damit unterstützen.

Der Unterschied zwischen Arbeitern und nicht arbeitenden Gettobewohnern hinsichtlich der Verpflegungssituation war groß: Anfang November 1940 ordnete Biebow an, dass Arbeitskräfte entsprechend größere Mengen an Lebensmitteln bekommen sollten, um ihre Leistungsfähigkeit zu erhalten. Dies ging zu Lasten der übrigen Bevölkerung, denn die dem Getto zugestandenen Rationen erhöhte die deutsche Verwaltung nicht. Seit Januar 1941 bekam jeder Gettobewohner 300 Gramm Brot am Tag, in den Ressorts genannten Fabriken beschäftigte Arbeiter dagegen $600 \mathrm{Gramm} .{ }^{10}$

Am Anfang fanden trotz der Bemühungen Rumkowskis und seiner Mitarbeiter bei weitem nicht alle „arbeitsfähigen“ Gettobewohner eine Anstellung. In dieser Zeit erreichten den Judenältesten oder sein „Sekretariat für Bittschriften und Beschwerden“ unzählige Briefe, deren Verfasser ihre Situation schilderten und um eine Arbeitsstelle baten. ${ }^{11}$

Durch Werbeaktionen von Hans Biebow und dadurch eingehende Bestellungen einerseits und Rumkowskis Organisationsgeschick andererseits wuchs die Produktion im Getto im Jahr 1941 dann aber trotz aller Schwierigkeiten beträchtlich. Vor allem seit dem Sommer stieg die Zahl der Arbeitskräfte sukzessi-

8 Julian Baranowski, The Łódź Ghetto 1940-1944/Łódzkie Getto 1940-1944, Vademecum, 2. verb. und erw. Aufl., Łódź 2003, S. 46-48; Peter Klein, Die „Gettoverwaltung Litzmannstadt““ 1940-1944. Eine Dienststelle im Spannungsfeld von Kommunalbürokratie und staatlicher Verfolgungspolitik, Hamburg 2009.

9 Chronik 1942, S. 148.

10 Löw, Juden, S. 127f.

11 Staatsarchiv Łódź, APŁ 278/158 sowie 278/200 bis 278/236. 
ve an. Immer mehr Aufträge erreichten das Getto. ${ }^{12}$ Im Dezember 1941 standen bereits gut 50000 Gettobewohner in einem Arbeitsverhältnis, darunter knapp 5000, die außerhalb des Gettos beim Bau der Autobahn von Frankfurt/Oder nach Posen beschäftigt waren. ${ }^{13}$ Der Jugendliche Dawid Sierakowiak beschreibt diese Entwicklung am 24. August 1941 in seinem Tagebuch:

Das Ghetto entwickelt sich immer prächtiger. Es entsteht eine Unmenge neuer Werkstätten und Fabriken, die zusammen mit den schon vorhandenen wahrhaftig den Jüdischen Industriebezirk bilden, wie wir scherzhaft sagen.

Und noch einer Aufzählung verschiedener Arbeitsstätten beschreibt er eines genauer:

Das Metall-Ressort entwickelt sich zusehends, es bekommt immer gewichtigere Aufträge von den Deutschen. Hunderte von Menschen finden Beschäftigung [...]..$^{14}$

In Krakau, der Hauptstadt des Generalgouvernements, war das deutsche Arbeitsamt („Arbeitsamt Abteilung Judeneinsatz“) in der ul. Józefińska 10 dafür zuständig, die Menschen im Getto auf verschiedene Arbeitsplätze zu verteilen. $\mathrm{Zu}$ diesem Zweck arbeitete es mit der Arbeitsamt-Abteilung des Judenrates zusammen - eine solche doppelte Verwaltung des jüdischen Arbeitseinsatzes gab es in vielen Gettos. ${ }^{15}$ Diese Judenratsabteilung wiederum führte eine Kartei der Gettobewohner, die nicht im Besitz einer Arbeitsbescheinigung waren. Die beruflichen Fähigkeiten der Juden waren hier verzeichnet, so dass sie gezielt zugeteilt werden konnten. Diejenigen ohne feste Anstellung wurden bei Bedarf verschiedenen Arbeiten zugeteilt. Sie mussten sich dazu morgens mit ihren Kennkarten beim Arbeitsamt melden. ${ }^{16}$

Keine großen Probleme hatten die zahlreichen Handwerker, jedoch: Für viele Krakauer Juden war es ungeheuer schwer, eine feste Arbeit zu finden,

12 Die Chronik des Gettos Lodz/Litzmannstadt 1941 (künftig: Chronik 1941), hrsg. v. Sascha Feuchert et al. Göttingen 2007, S. 227.

13 Tabelle über Beschäftigungszahlen: Henryk Rubin, Żydzi w Łodzi pod niemiecką okupacją 1939-1945, London 1988, S. 309.

14 Das Ghettotagebuch des David Sierakowiak. Aufzeichnungen eines Siebzehnjährigen 1941/42, Leipzig 1993, S. 82f.

15 Insgesamt hatte die deutsche Arbeitsverwaltung im Generalgouvernement bis zum Beginn des Massenmordes die Kompetenz für den Arbeitseinsatz der Juden inne; siehe Stephan Lehnstaedt, Die deutsche Arbeitsverwaltung im Generalgouvernement und die Juden, in: VfZ 60 (2012), S. 409-440.

$16 \mathrm{Zu}$ den Ausführungen zum Getto Krakau siehe: Andrea Löw/Markus Roth, Juden in Krakau unter deutscher Besatzung 1939-1945, Göttingen 2012. 
denn ihre Qualifikationen waren kaum mehr gefragt: Die Fabriken und Werkstätten suchten keine Lehrer, keine Anwälte und auch keine Schriftsteller oder Journalisten. Sie alle mussten nun unter Beweis stellen, dass sie zu körperlicher Arbeit in der Lage waren. Denn um auch nur mit dem nötigsten versorgt zu sein, musste man eine Arbeit finden. So kam es, wie Anna Lermer dies beschreibt, zu einem „fieberhaften Wettlauf zum Arbeitsamt“. ${ }^{17}$

Während die Arbeiter im Getto in Litzmannstadt sämtlich innerhalb des Stacheldrahtzaunes eingesetzt waren, verließen zahlreiche jüdische Arbeitskräfte des Krakauer Gettos dieses, um an ihre Arbeitsplätze zu gelangen. Verschiedenste Einsatzorte gab es außerhalb des Gettos für dessen Bewohner, sie waren etwa in der Kabelfabrik in Płaszów, dem Ziegelwerk „Bonarka“, am Flughafen in Rakowice (Fliegerhorstkommandatur) oder in der Emaillewarenfabrik von Oskar Schindler in der ul. Lipowa 4 beschäftigt. Zahlreiche deutsche und auch polnische Firmen profitierten von den billigen jüdischen Arbeitskräften. Jeden Morgen sammelten sich die Arbeiter vor dem Arbeitsamt, von wo aus sie in von deutschen, polnischen oder ukrainischen Wachen begleiteten Kolonnen zu ihren Arbeitsplätzen geführt wurden. Um das Getto zu verlassen, benötigten sie einen speziellen Passierschein, der sie als Arbeiter auswies. ${ }^{18}$

Es gab durchaus auch Juden, die in derselben Firma beschäftigt waren wie vor dem Krieg, manche dieser Firmen beschäftigten ihre jüdischen Mitarbeiter sogar kommissarisch weiter als Fachleute und Buchhalter bis zur ersten großen „Aussiedlungsaktion“ aus dem Getto im Juni 1942.

Auch auf dem Gettogelände selbst befanden sich Werkstätten, in denen Juden eingesetzt wurden, so die Textilfabrik des Österreichers Julius Madritsch. am Rynek Podgórski 2 und die Fabrik für optische Gläser des Polen Feliks Dziuba in der ul. Targowa 6. Dziuba war gleichzeitig Direktor der Firma „Spektrum“, die außerhalb des Gettos lag und Uhrengläser und Autoglasscheiben herstellte. Da beide Fabriken für die deutsche Wehrmacht arbeiteten, bekam Dziuba recht problemlos für sich und seine Angestellten Passierscheine.

Die Arbeitsbedingungen waren sehr unterschiedlich, ebenso variierte offenbar die Bezahlung, obwohl deren Höhe eigentlich von der deutschen Verwaltung vorgegeben war und seit Juli 1940 achtzig Prozent des Lohns für nichtjüdische Polen betragen sollte. Die Firmen überwiesen diese Löhne an den Judenrat, der einen Teil davon an die Arbeiter ausbezahlte, einen anderen Teil wie eine Steuer einbehielt und davon Fürsorgeleistungen für Bedürftige etc. finanzierte. Auch bekamen die Arbeiter zusätzliche Lebensmittel.

17 AŻ̇H, 301/2061, Bl. 4, Bericht Anna Lermer (Übersetzung aus dem Polnischen).

18 Löw/Roth, Juden, S. 75-82, auch für die folgenden Angaben. 
Manche organisierten ihre Arbeit selbst: Jüdische Handwerker schlossen sich zu Arbeitsgemeinschaften zusammen oder führten solche Verbünde aus der Zeit vor dem Krieg weiter. Sie organisierten Bestellungsannahme, Produktion und Auslieferung gemeinsam. Diese Zusammenschlüsse professionalisierten und vergrößerten die Produktion und machten die Handwerker damit für Kunden interessant. Der bereits 1940, also vor Errichtung des Gettos, ins Leben gerufene „Dachverband der Jüdischen Handwerker“ versuchte, erwerbslosen Juden wieder zu einer Arbeit zu verhelfen. In der ehemaligen Schokoladenfabrik „Optima“ gründeten jüdische Handwerker schon im Frühjahr 1941 eine Art Gemeinschaftswerkstatt. In zahlreichen Gettos gab es diese selbst organisierten Werkstätten. ${ }^{19}$

\section{Arbeit und Vernichtung}

Die Menschen im Getto versuchten also, eine Anstellung in einer der Werkstätten oder Fabriken zu finden, denn nur dies sicherte ihre, wenn auch karge, Versorgung mit Lebensmitteln. Mit dem Beginn der Deportationen in die Vernichtungslager Kulmhof und - im Fall von Krakau - Belzec bekam Arbeit in einem noch viel direkteren Sinne die Bedeutung, das Leben der Gettobewohner zu retten. Bis September 1942 ermordeten die Deutschen etwa 70000 Juden aus Litzmannstadt in Kulmhof. Die Auswahl der zu Deportierenden oblag zunächst Rumkowski. Zur Gruppe derer, die er und seine „Aussiedlungskommission“ zunächst auswählten, gehörten „Verbrecher“, also diejenigen Menschen, die vom Gericht wegen verschiedener Vergehen verurteilt worden waren oder im Zentralgefängnis einsaßen, und Gettobewohner, die nicht arbeiteten. Rumkowski musste in der Annahme, das Überleben des Gettos und möglichst vieler seiner Bewohner auf diese Weise zu sichern, die Prinzipien der Besatzer übernehmen und versuchte an erster Stelle die „arbeitsfähigen“ Juden $\mathrm{zu}$ retten. ${ }^{20}$

Aus dem Krakauer Getto wurden in zwei mehrtägigen Deportationsaktionen im Juni und Oktober 1942 rund 14000 Menschen nach Belzec deportiert und dort ermordet. Diese „Aktionen“ verliefen immer nach dem gleichen Schema: Deutsche und polnische Polizei umstellten das Getto, alle Gettobewohner muss-

$19 \mathrm{Zu}$ Beispielen aus anderen Orten als Krakau: Lehnstaedt, Arbeitsverwaltung, S. 426.

20 Löw, Juden, S. 265-268. Zu Rumkowski und seiner Strategie auch: Michal Unger, Reassessment of the Image of Mordechai Chaim Rumkowski, Jerusalem 2004; Monika Polit, Mordechaj Chaim Rumkowski. Prawda i Zmyślenie, Warszawa 2012. 
ten mit ihren Papieren zur Registrierung kommen, wo die Dokumente derjenigen gestempelt wurden, die zunächst von der Deportation ausgenommen werden sollten. Alle Übrigen mussten sich auf dem plac Zgody versammeln oder wurden unter roher Gewalt dorthin getrieben. Immer wieder waren Schüsse zu hören, die Menschen wurden getreten und geschlagen. Nahezu pausenlos brachten Helfer Verwundete ins Krankenhaus oder schafften Leichen fort. Die auf dem plac Zgody Zusammengetriebenen brachte man in einem Fußmarsch zum Bahnhof und zwängte sie in die bereitstehenden Züge. ${ }^{21}$

Von solcher Brutalität geprägt war auch die nächste große Deportationsaktion in Litzmannstadt im September 1942, die sog. „Sperre“: Mehr als 15000 Kinder unter zehn, ältere Menschen über 65 Jahren und Kranke wurden nach Kulmhof gebracht und dort ermordet. Nahezu jeder Gettobewohner verlor in diesen dramatischen Tagen Freunde und Verwandte. Die Gewalt, mit der die nun selbst ins Getto gekommene deutsche Polizei während der „Sperre“ agierte, und die Auswahl der Opfer führten dazu, dass die Ahnung über das Schicksal der Deportierten bei vielen Menschen im Getto zur Gewissheit wurde. So schrieb Oskar Singer in einer seiner Reportagen am 16. September 1942: „Alle sind fest davon überzeugt, dass man die ausgesiedelten Juden in die Vernichtung führt.“22 Und Oskar Rosenfeld notierte einige Tage nach dem Ende der „Sperre“: „Scheinbar alle ,Untaugl. 'Vergast etc., d.h. nicht mehr am Leben.“23

Umso wichtiger waren Überlebensstrategien - und die zentrale Strategie war Arbeit. Knapp 90000 Juden lebten noch im Getto, die Bevölkerungsstruktur hatte sich radikal verändert: Es gab kaum mehr junge, alte und kranke Menschen, außerdem waren im Zuge der Gettoauflösungen im Warthegau etwa 18000 „arbeitsfähige“ Juden nach Litzmannstadt gekommen. Die Bestellungen, die zuvor in diesen Gettos bearbeitet worden waren, gingen nun in das Getto Litzmannstadt. Lucjan Dobroszycki, der das Getto überlebt und später erforscht hat, konstatierte: „The ghetto had been turned into something like one great factory“. ${ }^{24}$ In einem Lagebericht der Gestapo Litzmannstadt vom 1. Oktober 1942 sind die Änderungen zusammengefasst:

$21 \mathrm{Zu}$ den Deportationsaktionen im Juni und Oktober 1942 siehe ausführlich Löw/Roth, Juden, S. S. $127-177$.

22 Oskar Singer, „Im Eilschritt durch den Gettotag...“ Reportagen und Essays aus dem Getto Lodz, hrsg. v. Sascha Feuchert et al., Berlin/Wien 2002, S. 134.

23 Oskar Rosenfeld, Wozu noch Welt: Aufzeichnungen aus dem Getto Lodz, hrsg. v. Hanno Loewy, Frankfurt a.M. 1994, S. 156.

24 Lucjan Dobroszycki: Introduction, in: ders.: The Chronicle of the Łódź Ghetto 1941-1944, New Haven/London 1984, S. ix-lxviii, S. lx. 
Durch diese letzte Aussiedlung ist der Bevölkerungsstand im Getto Litzmannstadt auf rund 89.500 Juden herabgesunken. Bei diesen Juden handelt es sich durchweg um arbeitsfähige Juden, die fast sämtlich in den Arbeitsprozess einbezogen worden sind. Der weit grössere Teil dieser arbeitenden Juden ist nach wie vor für die Ausfertigung von Wehrmachtsaufträgen eingesetzt, während ein kleinerer Teil mit der Ausfertigung von Privataufträgen beschäftigt wird. Bei den Privatauftraggebern handelt es sich um grössere Textil- und Schuhfirmen aus dem Altreichsgebiet. ${ }^{25}$

Mit dem Ende der „Sperre“ ordnete Hans Biebow am 12. September 1942 an, vom 14. September an die Arbeit in den Fabriken und Werkstätten wieder aufzunehmen. Er rief die Arbeiter dazu auf, ,pünktlich seinen Arbeitsplatz einzunehmen, wenn ihm daran gelegen ist, sich vor denkbar grössten Unannehmlichkeiten zu schützen“. Die Bekanntmachung beruhigte die Menschen, denn es war darin von „nunmehr anerkannten Arbeitskräfte[n]“26 die Rede. Dies deutet Oskar Rosenfeld in seinem Tagebuch an: „d.h. die Arbeitenden sind geschützt und werden ausreichend ernährt.“27

Die „Sperre“ hatte der jüdischen Bevölkerung vor Augen geführt, dass die aus dem Getto Deportierten vermutlich ermordet worden waren. Doch diejenigen, die im Getto geblieben waren, galten als Arbeitskräfte. Also, so der Schluss, betraf die Vernichtungspolitik der Nationalsozialisten „nur“ die nicht arbeitenden Juden. Rumkowskis von Beginn an verfolgter Leitspruch „Unser einziger Weg ist Arbeit“ hatte mit dem Einsetzen der Deportationen eine grundlegend neue Bedeutung bekommen: Jetzt war Arbeit der einzige zumindest temporäre Schutz vor dem Abtransport in die Vernichtung. Also arbeiteten die Menschen, und selbst die größten Kritiker der Politik des Judenältesten stimmten in der Annahme, dass Arbeit ihre einzige mögliche Rettung war, mit ihm überein. Oskar Rosenfeld schreibt Ende 1942 in sein Tagebuch: „Die deutschen Fachkommissionen (Fachleute, keine Politiker) finden Arbeit sehr gut, staunen über das Talent der Arbeiter. Getto wird durch Arbeit gesichert.“ Und wenige Tage später betont er noch einmal: „Getto als Arbeitslager bestätigt, daher Aussiedlung nicht in Frage, Lebensmittelnot nicht zu befürchten.“28

Ein anderer Gettobewohner schreibt, nun sei „ein neues, echtes Arbeitslager entstanden. Fast die ganze Bevölkerung des Gettos arbeitet, entsprechend dem Auftrag der Gettoverwaltung.“ Und er interpretiert die Vorgänge wie Ro-

25 Gestapo Litzmannstadt: AŻıH, 205/71, Lagebericht vom 1.10.1942.

26 Artur Eisenbach (Hrsg.), Dokumenty i Materiały do Dziejów Okupacji Niemieckiej w Polsce, Bd. III.: Getto Łódzkie, Warszawa 1946, S. 236: Getto-Verwaltung, gez. Biebow: Wiedereröffnung aller Fabriken und Werkstätten ab Montag, d. 14.9.1942 vom 12.9.1942.

27 Rosenfeld, Welt, S. 155.

28 Ebd., S. 174f. 
senfeld und vermutlich viele andere Menschen im Getto: „Wir werden arbeiten. Und arbeitsbereite Menschen wird man mit Essen versorgen!“ ${ }^{29}$ Niemand würde seine Arbeitskräfte ermorden - mit dieser Hoffnung lebten die Menschen nach den traumatischen Tagen der „Sperre“ weiter.

So verfolgte Rumkowski seine Strategie weiter und organisierte die Arbeit im Getto in einem noch größeren Ausmaß - forciert wurde diese Entwicklung wiederum von Biebow, der verstärkt eingriff. In ehemaligen Krankenhäusern, Schulen oder Waisenhäusern wurden neue Werkstätten eingerichtet, Arbeitsbedingungen verschärft und neue Aufträge, vor allem von der Wehrmacht, erreichten das Getto. Im Dezember 1942 waren 94 Fabriken in Betrieb, 87615 Menschen lebten im Getto, von denen 75650 beschäftigt waren; von den übrigen waren im Moment ungefähr 1200 krank und sollten danach wieder arbeiten. ${ }^{30}$

In Krakau erfolgte nach den Deportationen vom Juni und Oktober 1942 eine Aufteilung des Gettos: In Getto A lebten die Arbeitskräfte mit ihren Familien, in Getto B alle anderen. Die beiden Teile waren streng voneinander getrennt. Das Signal war deutlich: Arbeit ist überlebenswichtig. Ohnehin hatte die Organisation der Arbeit von Juden im gesamten Generalgouvernement eine grundlegende Änderung erfahren: Seit Juni 1942 durfte deren Einsatz nur noch im Einvernehmen mit den lokalen SS- und Polizeikräften geregelt werden. ${ }^{31}$

Bereits zu Beginn des Jahres 1942 waren immer mehr Arbeiter, vor allem Handwerker, dazu übergangen, sich zur Arbeit zusammenzuschließen, da sie sicher waren, dass nur ihr Status als Arbeitskraft sie vor der Deportation schützen würde. Und der neue Judenratsvorsitzende Dawid Gutter (der bisherige Vorsitzende Artur Rosenzweig war selbst deportiert und ermordet worden) verstärkte diese Bemühungen nach den Deportationen im Juni. Er gründete sogenannte Gemeinschaften von Schneidern, Tischlern usw. Die Mitglieder dieser Gemeinschaften produzierten für deutsche Abnehmer und, so hofften sie, machten sich dadurch unentbehrlich. Der Überlebende Zenon Szpingarn erinnert sich: „Die Sorge um die Zukunft war der hauptsächliche Grund für die massenhaften Bemühungen, in diesen Kooperativen Arbeit zu finden.“ ${ }^{32}$

Es gab weiterhin die Möglichkeit, außerhalb des Gettos einen Arbeitsplatz $\mathrm{zu}$ finden und Helmut Steinitz, der während der Deportationen seine Familie verloren hatte und nun auf sich gestellt im Getto lebte, erinnert sich: „Verschiedene Betriebe verteilten mittags eine warme Mahlzeit, damals ein ganz beson-

29 Arnfried Astel et al. (Hrsg.), Briefe aus Litzmannstadt, Köln 1967, S. 26 u. 31.

30 APE, GV 30024, Bl. 8, Rumkowski an Gettoverwaltung Litzmannstadt vom 4.1.1943.

31 Lehnstaedt, Arbeitsverwaltung, S. 415f.

32 AŻıH, 302/8, Bl. 22, Bericht Zenon Szpingarn (Übersetzung aus dem Polnischen). 
deres Privileg. Viele wurden in Militärgaragen als Automechaniker beschäftigt, ein Beruf, der mir interessanter erschien als Schlosser. Ich wollte in einer solchen Garage arbeiten, denn Arbeit außerhalb des Ghettos erschien mir eine gute Ablenkung für mich, und eine zusätzliche warme Mittagsmahlzeit war eine wichtige Hilfe in meiner kritischen Lage. “33

Mit Wirkung vom 3. Juni 1942 gingen im Generalgouvernement sämtliche „Judenangelegenheiten“ in die Zuständigkeit des Höheren SS- und Polizeiführers, Friedrich Wilhelm Krüger, über. Damit fiel auch der Arbeitseinsatz der jüdischen Bevölkerung in den Gettos von der deutschen Arbeitsverwaltung in den Kompetenzbereich des SS- und Polizeiapparates. Damit zusammenhängend ließ der zuständige SS- und Polizeiführer im Distrikt Krakau, Julian Scherner, neue Zwangsarbeitslager errichten, darunter auch das Lager Plaszow. ${ }^{34}$ Seit Dezember 1942 wurden Arbeiter, die außerhalb des Gettos beschäftigt waren, nach und nach in dieses neu errichtete Zwangsarbeitslager verlegt. Im Februar 1943 waren dort bereits 2000 Menschen eingesperrt. Im selben Monat wurde der Judenrat von der Absicht informiert, das Getto aufzulösen und die Arbeitskräfte ins Lager zu bringen. Im März 1943 wurde das Getto aufgelöst: Am 13. März mussten sich alle Bewohner von Getto A vor dem Gettotor einfinden, wo sie einer Selektion unterzogen und zum Beispiel alle Kinder zurückbehalten wurden. Die übrigen Juden führten die Deutschen ins Lager Plaszow. Am Tag darauf rückten SS- und Polizeikräfte in das Getto ein und trieben die Bewohner des Gettos B auf dem plac Zgody zusammen, wo sie stundenlang und unter ständiger Demütigung ausharren mussten. Dabei richteten die deutschen Einheiten im Getto ein Blutbad an. Die Patienten der Krankenhäuser erschossen sie in ihren Betten, auch diejenigen, die sie in Verstecken aufstöberten, töteten sie an Ort und Stelle. Auf dem Weg vom Getto zum Bahnhof erschossen die Begleitmannschaften immer wieder Einzelne. Insgesamt ermordeten sie an diesem Tag etwa 2000 Menschen im Getto oder auf dem Weg zum Zug, 3000 deportierten sie ins Vernichtungslager. Die Geschichte des Krakauer Gettos war zu Ende. ${ }^{35}$

33 Zwi Helmut Steinitz, Als Junge durch die Hölle des Holocaust. Von Posen durch Warschau, das Krakauer Ghetto, Płaszow, Auschwitz, Buchenwald, Berlin-Haselhorst, Sachsenhausen bis Schwerin und über Lübeck, Neustadt, Bergen-Belsen, Antwerpen nach Erez Israel 1927-1946, hrsg. v. Erhard Roy Wiehn, Konstanz 2006, S. 215.

34 Siehe zur Entwicklung im Distrikt Krakau insgesamt: Mario Wenzel, Die Umwandlung von Ghettos in Zwangsarbeitslager für Juden. Das Beispiel des Distrikts Krakau im Generalgouvernement 1942-1944, in: Hensel/Lehnstaedt, Arbeit, S. 361-373. Zu Plaszow siehe Ryszard Kotarba, Niemiecki obóz w Płaszowie 1942-1945, Warszawa/Kraków 2009.

35 Löw/Roth, Juden, S. 177-182. 
Das Jahr 1943 verlief im Getto Litzmannstadt dagegen relativ „ruhig“. Jedoch blieben die Lebens- und Arbeitsbedingungen auch in dieser Zeit stets lebensbedrohlich. Durch Hunger und Krankheit waren die Arbeiter in den Ressorts so geschwächt, dass sie die geforderte Leistung nicht immer erbringen konnten. Im Mai 1943 fand beispielsweise eine Beratung der Leiter der Schneidereien statt, die sich mit dem alarmierenden Problem beschäftigte, dass gerade unter den Arbeitern dieses wichtigsten Ressorts die Sterblichkeit zunahm. In der Tageschronik wurde am 21. Mai darüber berichtet und auf die enorme Bedeutung der Arbeit hingewiesen: „Die Beratung der Leiter galt der Aufgabe, einen Weg zur Abhilfe zu finden. Denn die wachsende Sterblichkeit unter den Ressortschneidern des Gettos kann für diese selbst katastrophale Folgen haben, sobald die Produktion der Schneider-Abteilungen unter das geforderte Mass fällt. Die Versorgung des Gettos mit Lebensmitteln haengt mit der Arbeitsleistung des Gettos zusammen. Wenn die von der deutschen Behörde erteilten Aufträge nicht im gewünschten Umfang und im zeitgerechten Termin ausgeführt werden, drohen dem Getto unausdenkbare Gefahren.“36

Lange sah es so aus, als würde Rumkowskis Strategie aufgehen. Doch Anfang 1944 änderte sich die deutsche Politik gegenüber dem Getto, und darauf hatten nun weder Rumkowski noch Biebow Einfluss: Gespräche über den Status des Gettos führten Anfang 1944 zu einer Übereinkunft zwischen Himmler und Gauleiter Greiser, dass das Getto zunächst verkleinert und später aufgelöst werden sollte. Spätestens im März 1944 befahl Himmler, das SS-Sonderkommando Kulmhof zu reaktivieren und Anfang Juni war es „betriebsbereit“. Am 23. Juni 1944 verließ der erste Transport den Bahnhof Radegast. ${ }^{37}$

An diesem Tag schreibt einer der Autoren der Tageschronik sehr hellsichtig über die Ambivalenz der Hoffnung, durch Arbeit der Vernichtung zu entgehen. In der Rubrik „Ressortnachrichten“ heißt es: „Am heutigen Tage kamen über Radegast beträchtliche Mengen von Rohwaren für die Schneiderbetriebe herein, worauf das Getto wieder /irrtümlich/ den Schluss zog, dass also das Getto nicht liquidiert werden dürfte. Natürlich hat eines mit dem andern nichts zu tun. Die Rohwaren sind ja längere Zeit unterwegs und gehören in den wirtschaftlichen Sektor des wirtschaftlichen Problems, das unabhängig vom politischen von anderen Stellen geleitet bzw. gelöst wird. “38

36 Die Chronik des Gettos Lodz/Litzmannstadt 1943 (künftig: Chronik 1943), hrsg. v. Sascha Feuchert, Göttingen 2007, S. 215f.

37 Michael Alberti, Die Verfolgung und Vernichtung der Juden im Reichsgau Wartheland 1939-1945, Wiesbaden 2006, S. 472-499.

38 Chronik 1943, S. 271. 
Der Schreiber sollte Recht behalten. Zwischen diesem 23. Juni und dem 14. Juli deportierten die Nationalsozialisten insgesamt 7196 Juden nach Kulmhof. Nach einem Deportationsstopp folgte dann die endgültige Auflösung des Gettos und knapp 70000 Menschen wurden aus dem Getto Lodz im August 1944 nach Auschwitz-Birkenau deportiert und die meisten dort sofort ermordet. Ungefähr 2000 der aus im Sommer deportierten Lodzer Juden wurden in das Lager aufgenommen und hatten damit eine Überlebenschance. Einige wurden noch weiter in andere Lager deportiert. Auf dem Gelände des Gettos blieb ein sogenanntes Aufräumkommando zurück, diese Gruppe bestand zunächst aus etwa 1300 Juden, von denen ungefähr 600 im Oktober zur Zwangsarbeit ins Deutsche Reich deportiert wurden. Die Gesamtzahl der Überlebenden aus dem Getto wird auf 5000 bis 7000 Menschen geschätzt. ${ }^{39}$

\section{Rettung durch Arbeit?}

Für die Menschen in den Gettos Litzmannstadt, Krakau und in vielen anderen Gettos war Arbeit ein zentraler Faktor in ihren Bemühungen zu überleben. Zunächst ging es darum, Geld und Lebensmittel zu erhalten, um sich und mitunter auch die eigene Familie versorgen zu können. Und auch für das Bemühen der Menschen in den Gettos, ihr Leben in der chaotischen und destruktiven Welt des Gettos zu ordnen, sich einen Alltag zu schaffen, spielte es eine wichtige Rolle, einer Arbeit nachzugehen. ${ }^{40}$ Seit Anfang 1942 konnte Arbeit dann in einem sehr wörtlich verstandenen Sinne das Leben retten, wurden doch in den allermeisten Fällen nur diejenigen Juden, die nachweisen konnten, dass sie Arbeitskräfte waren, von den Deportationen in die Vernichtung verschont. Doch war diese „Rettung durch Arbeit“ in vielen Fällen nur temporär. Viele derjenigen Juden in den Gettos, die durch ihre Arbeitskarten bei den ersten großen Deportationen noch verschont wurden, überlebten am Ende doch nicht. Selbst das Getto Litzmannstadt, das gewissermaßen sinnbildlich für den Versuch steht, die darin Eingeschlossenen durch ihre ungeheure Produktivität zu retten,

39 Alberti, Verfolgung, S. 493; Löw, Juden, S. 481-498.

40 Wie furchtbar dem gegenüber das Nichtstun war, beschreibt Oskar Rosenfeld kurz nach seiner Deportation in das Getto Litzmannstadt im Herbst 1941: „Hunde bellen, Pferde wiehern, Vögel zwitschern, Sklaven schufen, aber wir Menschen im Getto vegetieren, sich selbst zur Last. Wir haben den Sinn des Lebens verloren. Man braucht uns nicht erst auf den Mist zu werfen, wir liegen schon dort vom ersten Augenblick, an dem wir das Getto betreten haben. Das Nichtstun hat sich in unser Mark eingefressen und uns allmählich Abscheu vor allem eingegeben." Rosenfeld, Welt, S. 52. 
wurde am Ende vollständig aufgelöst und seine Bewohner in den meisten Fällen in den Tod deportiert. Die Überlebenden jedoch haben zumeist lange Zeit in den Gettos gearbeitet: Arbeit war für Juden eine notwendige, wenn auch keine hinreichende Bedingung, den Holocaust zu überleben.

Die Zeugnisse der Menschen in den Gettos machen deutlich, dass ihnen diese doppelte Bedeutung der „Rettung durch Arbeit“ sehr bewusst war und sie darum permanent versuchten, einen Arbeitsplatz zu finden.

Dieser Befund - Juden in den Gettos bemühten sich darum, Arbeit zu finden - war in den letzten Jahren von zentraler Bedeutung: Das im Juni 2002 einstimmig vom Deutschen Bundestag beschlossene „Gesetz zur Zahlbarmachung von Renten aus Beschäftigungen in einem Ghetto“ (ZRBG) sieht vor, dass in Gettos geleistete Arbeit unter bestimmten Bedingungen einen Rentenanspruch begründet. Etwa 70000 Anträge gingen daraufhin bei den Rentenversicherern ein, die jedoch zu etwa 90 Prozent abgelehnt wurden. Etwa ein Drittel der Abgelehnten ging vor die Sozialgerichte. Ohne an dieser Stelle in die Einzelheiten gehen zu können: Die Kläger mussten, um ihren Anspruch zu begründen, zwei Dinge belegen: Dass ihre Arbeit „angemessen“ entlohnt worden war und dass sie „aus eigenem Willensentschluss“ aufgenommen worden war. War Arbeit im Getto also keine Zwangsarbeit und angemessen entlohnt wurde sie auch? Und was bedeutet im Getto eine angemessene Entlohnung? Der Teller Suppe, der angesichts von Lebensmittelpreisen wichtiger war als der offizielle Lohn, kann nur schwer siebzig Jahre später nachgewiesen werden. Es wird deutlich, dass der Gesetzestext auf die extrem schwierigen Bedingungen in den Gettos im Grunde kaum anwendbar war. Diese Begrifflichkeiten des Versicherungsrechts bargen die Gefahr, die Leidenszeit der Menschen in den Gettos zu verharmlosen - so empfanden es auch zahlreiche Überlebende. Das große Ausmaß von Ablehnungen und die Art und Weise, wie hier teilweise argumentiert wurde, hat bei vielen Überlebenden zu großem Entsetzen geführt. ${ }^{41}$

Erst relativ spät, und leider eben auch nicht bei der Entstehung des Gesetzes, wurden Historiker hinzugezogen, dann jedoch in großem Umfang: Zahlreiche historische Gutachten wurden angefertigt, die die Bedingungen von Arbeit in den einzelnen Gettos und Regionen darstellten und mitunter - eigens zu diesem Zweck überhaupt erst detailliert erforschten. Unser Wissen über die Arbeit in den Gettos, die einen wichtigen Teil der Lebenswirklichkeit der Menschen ausmachte, hat sich dadurch in hohem Maße vergrößert. Viel genauer fragen Historikerinnen und Historiker in den letzten Jahren nach dem Leben der

41 Kristin Platt, Bezweifelte Erinnerung, verweigerte Glaubhaftigkeit. Überlebende des Holocaust in den Ghettorenten-Verfahren, München 2012. 
Menschen in den Gettos und untersuchen, wie diese versuchten, sich eine Art von Alltag unter den destruktiven Bedingungen $\mathrm{zu}$ schaffen, was zumindest teilweise in einem Zusammenhang mit der Gutachtertätigkeit steht. ${ }^{42}$

Anfang Juni 2009 hat dann das Bundessozialgericht darauf reagiert, dass die juristischen Begrifflichkeiten nicht dazu geeignet sind, die komplizierte Realität der Gettos angemessen zu erfassen, und hat in seiner Interpretation eine klare Abkehr von der bisher praktizierten Auslegung des ZRBG vollzogen. Der 5. Senat stellte am 3. Juni fest:

Die von der bisherigen Rechtsprechung aus den Voraussetzungen für eine rentenversicherungspflichtige Beschäftigung entwickelten Einschränkungen des Entgeltbegriffs bei Geringfügigkeit, Unangemessenheit oder freiem Unterhalt werden den tatsächlichen Lebensverhältnissen im Ghetto nicht gerecht; diese erfordern ein eigenes Verständnis des Entgeltbegriffs im Rahmen des ZRBG.

Außerdem stellte das Gericht fest, der Arbeitszwang für die jüdische Bevölkerung sei nicht mit Zwangsarbeit gleichzusetzen (die eben keinen Rentenanspruch begründen würde) und jegliche Form von Entlohnung, also auch die in Form von Lebensmitteln, sei anzuerkennen. Die historischen Erkenntnisse, die die Gutachten hervorgebracht hatten, sind offensichtlich in diese Argumentation eingeflossen. ${ }^{43}$

In diesem Fall hatten Historikerinnen und Historiker mit der Untersuchung eines nur scheinbar weit zurückliegenden Forschungsgegenstandes einen immens aktuellen Bezug. Und für zahlreiche derjenigen Juden, die vermutlich auch aufgrund ihrer Arbeit in den Gettos überhaupt erst überlebten, bleiben diese Fragen aktuell. Zahlreiche Verfahren wurden nach den wichtigen Ent-

42 Um diese Forschungen und Befunde zu bündeln, fand im Dezember 2010 eine Tagung im Deutschen Historischen Institut in Warschau zur Frage der Arbeit in den Gettos statt; siehe den Tagungsbericht auf [http://hsozkult.geschichte.hu-berlin.de/tagungsberichte/id=3544], eingesehen 26.1.2014. Der Band ist gerade erschienen: Hensel/Lehnstaedt, Arbeit. Schon 2008 hatte im Institut für Zeitgeschichte die von Jürgen Zarusky organisierte Konferenz „Ghettorenten und historische Forschung“ versucht, vor allem die beteiligten Richter und Historikerinnen und Historiker zusammen zu bringen; Jürgen Zarusky, Ghettorenten. Entschädigungspolitik, Rechtsprechung und historische Forschung, München 2010. Zur Frage des Alltags: Andrea Löw/ Doris L. Bergen/Anna Hájková (Hrsg.), Alltag im Holocaust. Jüdisches Leben im Großdeutschen Reich 1941-1945, München 2013 sowie der angekündigte Tagungsbad: Imke Hansen et al. (Hrsg.), Lebenswelt Ghetto. Alltag und soziales Umfeld während der nationalsozialistischen Verfolgung, Wiesbaden 2013.

43 Zit. n.: Jürgen Zarusky, Einleitung, in: ders., Ghettorenten, S. 8. Siehe auch Stephan Lehnstaedt, Geschichte und Gesetzesauslegung. Zu Kontinuität und Wandel des bundesdeutschen Wiedergutmachungsdiskurses am Beispiel der Ghettorenten, Osnabrück 2011. 
scheidung des Bundessozialgerichts wieder aufgenommen. Anfang 2012 waren von knapp 50000 Fällen, die die Rentenversicherer noch einmal überprüfen mussten, etwa die Hälfte zu Gunsten der Kläger entschieden. Doch haben diese mehrere Jahre verloren - mehrere Tausend waren inzwischen bereits verstorben - und die Zahlungen sollen auch nur rückwirkend ab 2005 und nicht, wie im Gesetz ursprünglich vorgesehen, ab der ersten diesbezüglichen Grundsatzentscheidung des BSG im Jahre 1997, erfolgen. Dies hat das Bundessozialgericht im Februar 2012 nochmals bestätigt. Der Bundestag hat im März 2013 die Änderung der Rückwirkungspraxis abgelehnt, ebenso eine Entschädigungslösung, die ebenfalls im Gespräch war. ${ }^{44}$

Bei ihrem Israel-Besuch im Mai 2013 jedenfalls musste Justizministerin Sabine Leutheusser-Schnarrenberger konstatieren, dass es in der Frage der Gettorenten weiterhin Handlungsbedarf gebe: „Das ist eine Verantwortung, der wir unbedingt nachkommen müssen.“ ${ }^{45}$ Viel Zeit bleibt nicht: Die wenigen noch lebenden Juden, die in den nationalsozialistischen Gettos schufteten, sind hochbetagt.

44 Stephan Lehnstaedt, Wiedergutmachung im 21. Jahrhundert. Das Arbeitsministerium und die Ghettorenten, in: VfZ 3 (2013), S. 363-390. Hier auch aktuelle Zahlen zu nach dem Grundsatzurteil 2009 eingegangenen Anträgen und eine detaillierte Rekonstruktion des jahrelangen Gezerres um die Gettorenten.

45 Zit. n.: http://www.europeonline-magazine.eu/leutheusser-schnarrenbergerhandlungsbedarf-bei-ghetto-renten_282147.html, eingesehen 26.1.2014. 